\title{
Acurácia de dias de avaliação da gravidade especifica como medida da qualidade de
}

\section{casca de ovos de galinhas}

\author{
Accuracy of specific gravity assessment days as a measure of layer egg shell quality \\ Exactitud de los días de evaluación de la gravedad específica como medida de la calidad de la \\ cáscara del huevo de gallina
}

Recebido: 17/04/2021 | Revisado: 25/04/2021 | Aceito: 26/04/2021 | Publicado: 11/05/2021

Túlio Leite Reis

ORCID: https://orcid.org/0000-0003-2141-8740 Universidade Federal Rural do Rio de Janeiro, Brasil E-mail: tulioreis@hotmail.com

José Evandro de Moraes

ORCID: https://orcid.org/0000-0002-9105-6661 Instituto de Zootecnia, Brasil

E-mail: evandro.moraes@sp.gov.br

Ligia Fatima Lima Calixto

ORCID: https://orcid.org/0000-0002-6448-0643 Universidade Federal Rural do Rio de Janeiro, Brasil E-mail:1flcalixto@uol.com.br

Carla Cachoni Pizzolante

ORCID: https://orcid.org/0000-0002-4992-5982 Instituto de Zootecnia, Brasil

E-mail: carla.pizzolante@sp.gov.br

\begin{abstract}
Resumo
A análise de gravidade específica (GE) é o principal método indireto para mensurar a qualidade de casca de ovos de galinhas e largamente utilizado em pesquisas e pelo setor produtivo. No entanto verifica-se falta de padronização da quantidade de dias que geralmente são utilizadas para realização desta avaliação. Além disso, a temperatura ambiente pode modificar ao longo dos dias de avaliação da GE e, ocorrer elevação, o ovo poderá perder água e dióxido de carbono e, alterar a densidade do conteúdo interno, mascarando os resultados e interferindo na acurácia do mesmo. Portanto o objetivo desse trabalho foi verificar a quantidade de dias necessários para avaliar a qualidade de ovos através da GE. Utilizou-se um delineamento inteiramente casualizado, em esquema fatorial $3 \times 7$ (três dias de avaliação de GE, e sete soluções salinas). Os resultados obtidos foram submetidos à análise de variância e, quando significativos, ao teste de Tukey a 5\%. Não foram observadas diferenças significativas ( $p>0,05)$ para GE nos três dias avaliados, mas diferenças foram observadas $(\mathrm{p}<0,05)$ entre as percentagens de ovos que flutuaram nas sete soluções salinas e, de acordo com a equação de regressão obtida, conforme os valores de GE aumentaram, a quantidade de ovos de poedeiras semipesadas que flutuaram nessas densidades também aumentou. Portanto, pode-se concluir que, apenas um dia de avaliação da GE é suficiente para determinar com segurança e acurácia a qualidade de casca.
\end{abstract}

Palavras-chave: Método de imersão em solução salina; Método indireto; Qualidade ovos.

\begin{abstract}
Specific gravity analysis (SG) is the main indirect method for measuring the quality of hens' eggshells and is widely used in research and by the productive sector.However, there is not standardization in the number of days that are generally used to carry out this assessment. In addition, the ambiental temperature may change over the SG evaluation days and, if elevated, the egg may lose water and carbon dioxide and alter the density of the internal content, masking the results and interfering with the accuracy of the same. Therefore, the objective of this work was to verify the number of days necessary to evaluate the quality of eggs through SG. A completely randomized design was used, in a $3 \times 7$ factorial scheme (three days of SG evaluation, and seven saline solutions). The results obtained were submitted to analysis of variance and, when significant, to the Tukey test at $5 \%$. No significant differences were observed ( $p>0.05)$ for SG in the three days evaluated, but differences were observed $(p<0.05)$ between the percentages of eggs that fluctuated in the seven saline solutions and, According to the regression equation obtained, as the GE values increased, the amount of semi-heavy laying eggs that fluctuated in these densities also increased. Therefore, it can be concluded that just one day of GE evaluation is sufficient to safely and accurately determine the quality of the shell.
\end{abstract}

Keywords: Saline immersion method; Indirect method; Quality eggs. 


\begin{abstract}
Resumen
El análisis de gravedad específica (GE) es el principal método indirecto para medir la calidad de la cáscara de huevo de gallina y es ampliamente utilizado en la investigación y por el sector productivo. Sin embargo, existe una falta de estandarización en el número de días que generalmente se utilizan para realizar esta evaluación. Además, la temperatura ambiente puede cambiar durante los días de evaluación de GE y, si se eleva, el huevo puede perder agua y dióxido de carbono y cambiar la densidad del contenido interno, enmascarando los resultados e interfiriendo con la precisión de los mismos. Por lo tanto, el objetivo de este trabajo fue verificar la cantidad de días necesarios para evaluar la calidad de los huevos a través de GE. Se utilizó un diseño completamente al azar, en un esquema factorial 3x7 (tres días de evaluación de GE y siete soluciones salinas). Los resultados obtenidos se sometieron a análisis de varianza y, cuando fueron significativos, a la prueba de Tukey al 5\%. No se observaron diferencias significativas (p> $0.05)$ para EG en los tres días evaluados, pero sí se observaron diferencias $(p<0.05)$ entre los porcentajes de huevos que fluctuaron en las siete soluciones salinas y, según la ecuación de regresión obtenida, como Los valores de GE aumentaron, la cantidad de huevos de puesta semi-pesados que fluctuaron en estas densidades también aumentó. Por lo tanto, se puede concluir que un solo día de evaluación de GE es suficiente para determinar de manera segura y precisa la calidad del peeling.
\end{abstract}

Palabras clave: Método de inmersión salina; Método indirecto; Calidad de huevos.

\title{
1. Introdução
}

A casca do ovo é composta, principalmente, de carbonato de cálcio, com pequenos poros para a troca de gases, servindo de proteção contra os danos físicos e contaminantes (Silva et al., 2020). São vários os fatores que alteram a formação da casca de forma direta ou indireta (Carvalho et al., 2016). A avaliação da qualidade da casca de ovos pode ser realizada por métodos diretos, que embora sejam de fácil realização necessitam de equipamentos como balanças, micrômetros, além de serem são métodos onde o produto é perdido não sendo possível sua comercialização, o que torna essas metodologias mais restritas à áreas de pesquisa. Portanto métodos não destrutivos de avaliação (indiretos), como análise de gravidade específica (GE), são os mais utilizados na indústria por serem rápidos, práticos, baratos, e não necessitarem de quebra ou dano ao ovo, que pode ser aproveitado comercialmente (Jácome et al., 2012).

O primeiro pesquisador a relatar que a gravidade específica dos ovos pode ser utilizada como método de determinação da qualidade da casca foi Olsson (1934), citados por Freitas et al. (2004). A GE apresenta relação direta com o percentual de casca, pois os valores de densidade da gema e do albúmen em ovos frescos é muito próxima à densidade da água, então quando os ovos são imersos em soluções de menor densidade e na sequência, passando para as de maior densidade, a solução em que o ovo flutuar corresponderá a gravidade determinada, portanto quanto maior a gravidade especifica melhor será a qualidade da casca (Olsson, 1934).

As análises de resistência à quebra e espessura da casca dos ovos possuem correlação positiva com a GE, demonstrando a eficiência desse método para avaliar a qualidade da casca de ovos de galinha (Reis et al., 2019).

O conteúdo interno dos ovos tem densidade próxima à da água, assim, é possível mensurar a densidade da casca mergulhando os ovos em soluções salinas com densidades variadas. A GE pode ser mensurada, utilizando o método da imersão em solução salina (ISS), preparadas em baldes plásticos, contendo as diferentes soluções salinas ( $\mathrm{NaCl})$.

As densidades variam entre 1,060 e 1,100 g/ $\mathrm{cm}^{3}$, em intervalos de $0,005 \mathrm{~g} / \mathrm{cm}^{3} \mathrm{e}$, devem ser calibrados com auxílio de um densímetro. Os ovos a serem submergidos devem ser frescos e coletados logo após a postura, com um intervalo máximo de 3 horas entre a postura do primeiro e do último ovo que serão analisados, a fim de se padronizar as amostras no dia da avaliação, para que não ocorra interferência de tempo de postura, seguindo recomendação de Hamilton (1982).

Inicia-se mergulhando os ovos nos baldes contendo a solução de menor densidade $(1,060)$, se afundarem deve-se mergulhá-los na solução seguinte e assim sucessivamente, até que flutuem na solução de maior concentração de acordo com a metodologia proposta por Castelló et al. (1989). A gravidade específica (ou densidade da casca) será igual à solução em que o ovo flutuar.

Considera-se que quanto maior a gravidade específica, maior densidade da casca e, portanto, melhor a resistência. 
Dessa forma, valores acima de 1,085 g/ $\mathrm{cm}^{3}$ são considerados satisfatórios (Peebles \& McDaniel, 2004).

Segundo Abdallah et al. (1993), existe redução na quantidade de ovos quebrados com o aumentos da GE com alta correlação negativa $(\mathrm{r}=-0,96)$. Segundo os mesmos autores, a cada aumento de $0,001 \mathrm{~g} / \mathrm{cm} 3$ na gravidade específica, existe uma redução de $1,266 \%$ na porcentagem de ovos quebrados.

No entanto, essa metodologia de avaliação de qualidade de casca pode apresentar alguns desafios, principalmente porque pode ocorrer influencia da temperatura das soluções salinas, evaporação da água e erros relacionados com a calibração e leitura incorreta do equipamento usado para medir a densidade da solução salina (densímetro). Fissuras na casca do ovo, a água e a movimentação das soluções durante a condução da análise, também podem afetar o resultado final (Voisey \& Hamilton, 1977).

Além dessas intercorrências, a literatura consultada tem indicado uma série de pesquisas, utilizando uma determinada quantidade de dias consecutivos, para obtenção da GE, geralmente, três dias consecutivos. Contudo, com o passar do tempo, principalmente em dias de temperatura elevada, o ovo pode perder água e dióxido de carbono, alterando assim a densidade do conteúdo interno, o que pode mascarar os resultados esperados. Em função do exposto, objetivou-se com o presente trabalho verificar quantos dias são necessários para mensurar a gravidade específica de ovos e obter a acurácia dos resultados.

\section{Metodologia}

A GE foi avaliada, coletando-se ovos de poedeiras semipesadas, ao longo de quatro períodos de 28 dias cada, durante três dias consecutivos. A primeira coleta de ovos foi realizada quando as aves estavam com 25 semanas de idade e, a última coleta ao final do quarto período, quando as aves estavam com 37 semanas de idade.

A metodologia consistiu em retirar e descartar os ovos postos à noite a durante a madrugada, seguindo-se a coleta dos ovos que eram postos em seguida e transportados e avaliados imediatamente no laboratório. A avaliação da GE foi feita com a imersão sequencial dos ovos em baldes plásticos contendo sete diferentes soluções salinas de acordo com a metodologia proposta por Castelló et al. (1989), com as densidades variando entre 1.070 e 1.100 e intervalos de $0,005 \mathrm{~g} / \mathrm{cm}^{3}$, calibrados com densímetro.

Foram anotadas a quantidade de ovos que flutuavam nas sete diferentes soluções salinas, processo este que foi repetido durante três dias consecutivos, sempre ao final de cada período de 28 dias.

No total foram coletados 2174 ovos (média do primeiro dia referente a quatro períodos de 28 dias cada), 2323 ovos no segundo dia (média do segundo dia referente a quatro períodos de 28 dias cada) e 2331 ovos no terceiro dia (média do terceiro dia referente a quatro períodos de 28 dias cada), num total de 6828 ovos. Utilizou-se um delineamento inteiramente casualizado, em esquema fatorial $3 \times 7$ (três dias de avaliação de GE e sete soluções salinas com densidades variando entre 1.070 e $1.100 \mathrm{~g} / \mathrm{cm}^{3}$ e intervalos de $0,005 \mathrm{~g} / \mathrm{cm}^{3}$, calibrados com densímetro).

Os ovos quando mergulhados nas soluções salinas de menor densidade, afundavam, dessa forma, eram imersos na solução seguinte de maior concentração de sal e assim sucessivamente até que flutuassem.

A gravidade específica (ou densidade da casca) obtida era igual à solução de menor densidade em que os ovos flutuaram. Ao final, comparou-se a quantidade de ovos em percentagem que flutuou nas sete soluções salinas, nos três dias consecutivos, dos quatro períodos avaliados.

Os resultados quantitativos (Pereira et al., 2018) obtidos foram submetidos à análise estatística através da ANOVA e o contraste entre médias de tratamentos, quando significativos, ao teste de Tukey a 5\%, com auxílio do pacote computacional SISVAR (Ferreira, 2011). 


\section{Resultados e Discussão}

Os valores médios da percentagem de ovos que flutuaram nas diferentes soluções salinas para avaliação da gravidade específica durante três dias consecutivos são apresentados na Tabela 1.

Não foram observadas diferenças significativas $(p>0,05)$ da GE de ovos dentro da mesma solução salinas, quando comparados os três dias de avaliação, mas diferenças significativas $(\mathrm{p}<0,05)$ foram observadas entre as percentagens de ovos que flutuaram nas diferentes soluções salinas. De acordo com a equação de regressão obtida, conforme os valores de GE aumentaram, a quantidade de ovos que flutuaram nessas densidades também aumentou, sendo a grande maioria observada na faixa considerada satisfatória para ovos frescos (acima de 1,080), de acordo com Peebles e McDaniel (2004).

Tabela 1. Percentagem de ovos que flutuaram nas diferentes soluções salinas na avaliação da gravidade específica durante três dias consecutivos.

(\%) Ovos que flutuaram nas diferentes densidades

\begin{tabular}{|c|c|c|c|c|}
\hline \multirow{3}{*}{$\begin{array}{l}\text { Soluções salinas } \\
\end{array}$} & \multicolumn{3}{|c|}{ (\%) Ovos que flutuaram nas diferentes densidades } & \multirow[b]{3}{*}{ Média } \\
\hline & \multicolumn{3}{|c|}{ Dias de avaliação } & \\
\hline & 1 & 2 & 3 & \\
\hline 1,070 & 0,08 & 0,06 & 0,05 & $0.06 f$ \\
\hline 1,075 & 0,08 & 0,06 & 0,05 & $0.06 \mathrm{f}$ \\
\hline 1,080 & 2,33 & 2,57 & 2,56 & $2.47 \mathrm{e}$ \\
\hline 1,085 & 7,22 & 6,80 & 6,97 & $7.00 \mathrm{~d}$ \\
\hline 1,090 & 19,10 & 18,52 & 18,52 & $18.71 \mathrm{c}$ \\
\hline 1,095 & 31,35 & 32,31 & 32,66 & $32.11 \mathrm{~b}$ \\
\hline 1,100 & 39,16 & 38,66 & 38,19 & $38.67 \mathrm{a}$ \\
\hline Média & 14.18 & 14.14 & 14.14 & 14.16 \\
\hline CV (\%) & \multicolumn{4}{|c|}{0,52} \\
\hline Equação de regressão & \multicolumn{4}{|c|}{$Y=-1506,11913790+1,40117440 X$} \\
\hline $\mathrm{R}^{2}$ & \multicolumn{4}{|c|}{$89,87 \%$} \\
\hline
\end{tabular}

Médias seguidas de letras iguais não diferem $(\mathrm{p}>0,05)$ entre si nas colunas pelo teste Tukey. Fonte: Autores.

O maior número de ovos que flutuaram nas maiores densidades pode estar relacionado a vários fatores que exercem influência na qualidade dos ovos, como a idade da poedeira. A medida que a galinha envelhece, ocorre aumento do tamanho do ovo, e esse por sua vez não tem disponível a mesma quantidade proporcional de carbonato de cálcio para formação da casca, portanto ocorre piora da qualidade dessa estrutura, resultando na queda nos valores de GE (Carvalho \& Fernandes, 2012). No presente estudo as aves são consideradas jovens, 37 semanas no momento da realização do método, consequentemente, com melhor qualidade de casca.

A temperatura e umidade relativa do ar são fatores que exercem influência sobre a GE, assim, ovos armazenados por mais tempo em condições ambientais desfavoráveis, têm sua GE piorada (Saccomani et al., 2019). Os ovos deste estudo foram considerados frescos, pois eram coletados e avaliados logo após à postura, dificultando dessa forma a interferência de fatores adversos decorrentes do armazenamento. De acordo com Barbosa et al. (2008), a densidade e a massa são grandezas diretamente proporcionais, portanto utilizando a fórmula para o cálculo da densidade $(\mathrm{d}=$ massa/volume), quando ocorre decréscimo na massa, simultaneamente, ocorre decréscimo na densidade. Consequentemente, a menor perda de massa resulta em maiores valores de GE, como pode ser observado nos resultados obtidos para os ovos de poedeiras semipesadas (Tabela 1). 
O tempo decorrido após a postura provoca uma perda progressiva de água e dióxido de carbono através da casca, promovendo aumento do $\mathrm{pH}$ do ovo e redução do seu conteúdo. Quanto maior esse tempo, maior a perda intensificando a contínua retração da membrana interna da casca do ovo (aquela que está em contato com a albumina) e aumento do espaço entre esta e a membrana externa (aquela que está em contato com a casca do ovo). Este espaço é denominado de câmara de ar. Quanto mais fresco o ovo, menor ele é, pois quase não houve perda do conteúdo interno, e portanto maior a densidade. Então, a densidade total do ovo fresco é maior do que a do ovo mais velho, porque ele, contem maior volume ocupado por gás que reduz consideravelmente a densidade total (Sarcinelli et al. 2007; Santos et al. 2009).

Não ocorreram diferenças significativas ( $p>0,05)$ entre as análises de gravidade específica realizadas em um, dois ou três dias consecutivos, indicando que mantidas as condições ambientais, se nos dias consecutivos após o primeiro dia de analise, ocorreram quaisquer interferências capazes de afetar a gravidade especifica estas não foram relevantes e nem suficientes para alterar os resultados obtidos no primeiro dia, portanto apenas um dia de analise foi eficaz para determinar com acurácia da GE dos ovos produzidos no lote utilizado no ensaio experimental, dispensando a repetição em dias consecutivos, que demandaria tempo, maior volume de ovos e mais mão de obra.

\section{Conclusão}

Nas condições em que foi realizado o estudo e de acordo com os resultados obtidos, pode-se concluir que apenas um dia de avaliação de gravidade específica pelo método de imersão em solução salina é suficiente para determinar com segurança e acurácia a qualidade de casca, dispensando a repetição em dias consecutivos, que demandaria tempo, maior volume de ovos e mão de obra.

Novos estudos avaliando a variações de temperaturas entre os dias de análise de GE são recomendadas, visando averiguar possíveis alterações nos resultados.

\section{Referências}

Abdallah, A. G., Harms, R. H. \& El-Husseiny, O. (1993). Various methods of measuring shell quality in relation to percentage of cracked eggs. Poultry Science, 72(11), 2038-2043.

Barbosa, N. A. A., Sakomura, N. K., Mendonça, M. D. O., Freitas, E. R. \& Fernandes, J. B. K. (2008). Qualidade de ovos comerciais provenientes de poedeiras comerciais armazenados sob diferentes tempos e condições de ambientes. Ars Veterinaria, 24(2), $127-133$.

Carvalho, L. S. S. \& Fernandes, E. A. (2013). Formação e qualidade da casca de ovos de reprodutoras e poedeiras comerciais. Medicina Veterinária (UFRPE), 7(1), 35-44.

Carvalho, L. S. S., Vilela, D. R., Fagundes, N. S., Souza, Y. L. S. \& Fernandes, E. D. A. (2016). Qualidade de ovos e desempenho produtivo de poedeiras em segundo ciclo de postura alimentadas com microminerais quelatados a aminoácidos. Ciência Animal Brasileira, 17(4), 491-500.

Castelló, J. A., Pontes, M. \& González, F. F. (1989). Producción de huevos. Real Escuela de Avicultura. Barcelona, España.

Ferreira, D. F. (2011). Sisvar: a computer statistical analysis system. Ciência e agrotecnologia, 35(6), 1039-1042.

Freitas, E. R., Sakomura, N. K., Gonzalez, M. M. \& Barbosa, N. A. A. (2004). Comparação de métodos de determinação da gravidade específica de ovos de poedeiras comerciais. Pesquisa Agropecuária Brasileira, 39(5), 509-512.

Hamilton, R. M. G. (1982). Methods and factors that affect the measurement of egg shell quality. Poultry science, 61(10), 2022-2039.

Jácome, I. M. D. T., Borille, R., Rossi, L. A., Rizzotto, D. W., Becker, J. A. \& Sampaio, C. (2012). Desempenho produtivo de codornas alojadas em diferentes sistemas de iluminação artificial. Archivos de zootecnia, 61(235), 449-456.

Olsson, N. (1934). Studies on Specific Gravity of Hens' Eggs: A New Method for Determining the Percentage of Shell in Hens' Eggs. Otto Harrassowitz.

Peebles, E. D. \& McDaniel, C. D. (2004). Practical manual for understanding the shell structure of broiler hatching eggs and measurements of their quality. Missippi Agric. \& Forestry Experiment Station.

Pereira, A. S., Shitsuka, D. M., Parreira, F. J. \& Shitsuka, R (2018). Metodologia da pesquisa científica. Santa Maria/RS. Ed. UAB/NTE/UFSM.

Reis, T. L., Quintero, J. C. P., Moraes, J. E., Pizzolante, C. C. \& Calixto, L. F. L. (2019). Correlation among the main parameters of eggshell quality analysis of hen and quail eggs. Boletim De Indústria Animal, 76, 1-6. 
Research, Society and Development, v. 10, n. 5, e40410515148, 2021

(CC BY 4.0) | ISSN 2525-3409 | DOI: http://dx.doi.org/10.33448/rsd-v10i5.15148

Saccomani, A. P. O., Moraes, J. E., Reis, T. L., Ganeco, A. G., Thimotheo, M., Borba, H., Calixto, L. F. L. \& Pizzolante, C. C. (2019). Indicadores da qualidade fisico-química de ovos de poedeiras semipesadas criadas em diferentes sistemas de produção. Boletim De Indústria Animal, 76, 1-15.

Santos, M. D. S. V. D., Espindola, G. B., Lôbo, R. N. B., Freitas, E. R., Guerra, J. L. L. \& Santos, A. B. E. (2009). Effect of temperature and storage of eggs. Ciência e Tecnología de Alimentos, 29(3), 513-517.

Sarcinelli, M. F., Venturini, K. S. \& Silva, L. C. (2007). Características dos ovos. Universidade Federal do Espírito Santo.

Silva, W. C., Araújo, L. N., da Silva, É. B. R., de Sousa, E. D. V., da Cruz Gato, A. P. \& da Silva, J. Á. R. (2020). Revisão sistemática e cienciometria da produção de ovos comerciais no Brasil. Research, Society and Development, 9(10), e1399108459-e1399108459.

Thompson, B. K. \& Hamilton, R. M. G. (1982). Comparison of the precision and accuracy of the flotation and Archimedes' methods for measuring the specific gravity of eggs. Poultry Science, 61(8), 1599-1605.

Voisey, P. W. \& Hamilton, R. M. G. (1977). Sources of error in egg specific gravity measurements by the flotation method. Poultry science, 56(5), 1457-1462. 